\title{
The electron acceleration at shock waves in the solar corona
}

\author{
R. Miteva and G. Mann
}

\author{
Astrophysikalisches Institut Potsdam, An der Sternwarte 16, 14482 Potsdam, Germany \\ e-mail: rmiteva@aip.de
}

Received 1 December 2006 / Accepted 26 July 2007

\begin{abstract}
Context. In the solar corona, shock waves are generated by flares and/or coronal mass ejections. They are able to accelerate electrons up to high energies and can thus be observed as type II bursts in the nonthermal solar radio radiation. In-situ measurements of shock waves in interplanetary space have shown that shock waves attached by whistler waves are preferably accompanied by the production of energetic electrons.

Aims. Motivated by these observations, we study the interaction of electrons with such whistlers, which are excited by the protons accelerated by the shock.

Methods. We start with a resonant whistler wave-proton interaction that accounts for the initial whistler wave generation. Then, we consider resonant whistler wave-electron interaction, treated with a relativistic approach that is responsible for the electron energization in the whistler wave field.

Results. As a result, we show that electrons can be accelerated by a resonant wave particle (i.e., whistler-electron) interaction. This mechanism acts in the case of quasi-perpendicular shock waves. After acceleration, the energetic electrons are reflected by the associated shock wave back into the upstream region. The theoretical results are compared with observations, e.g., solar type II radio bursts.
\end{abstract}

Key words. shock waves - acceleration of particles - Sun: flares - Sun: radio radiation

\section{Introduction}

Shock waves play an important role in astrophysics, since they are responsible for particle acceleration in space plasmas (Fermi 1949; Axford et al. 1978; Schlickeiser 1984; Kirk 1994). The production of energetic electrons at shocks is of special interest, since these electrons can be the source of nonthermal radio and X-ray radiation, which can be observed by remote sensing techniques.

In the solar corona, shock waves can be generated either by blast waves due to the flare process (Uchida et al. 1973; Vrsnak et al. 1995) and/or by coronal mass ejections (CMEs) (Stewart et al. 1974a,b; Gopalswamy \& Kundu 1992; Classen \& Aurass 2002; Lara et al. 2003). It is still an ongoing debate whether the shock waves are generated by a flare-associated blast wave (Vrsnak et al. 1995) or if they are CME-driven (Gopalswamy \& Kundu 1992; Gopalswamy 2006). But it should be emphasized that a shock wave is a discontinuity accompanied by an increase in the entropy. The jump in the density, temperature, and magnetic field across the shock is governed by the Rankine-Hugoniot relationships (Priest 1982); i.e., the shock itself and the processes immediately associated with it should be considered as independent of its exciter.

In the corona, shock waves can be observed as type II bursts in the solar radio radiation (Wild \& McCready 1950; Uchida et al. 1973). A type II radio burst can morphologically be divided into two types of emission in its dynamic radio spectrum (see Fig. 1, for instance), the "backbone" and the "herringbone" emission. The so-called backbone emission (see Fig. 1), is slowly drifting from high to low frequencies with a typical

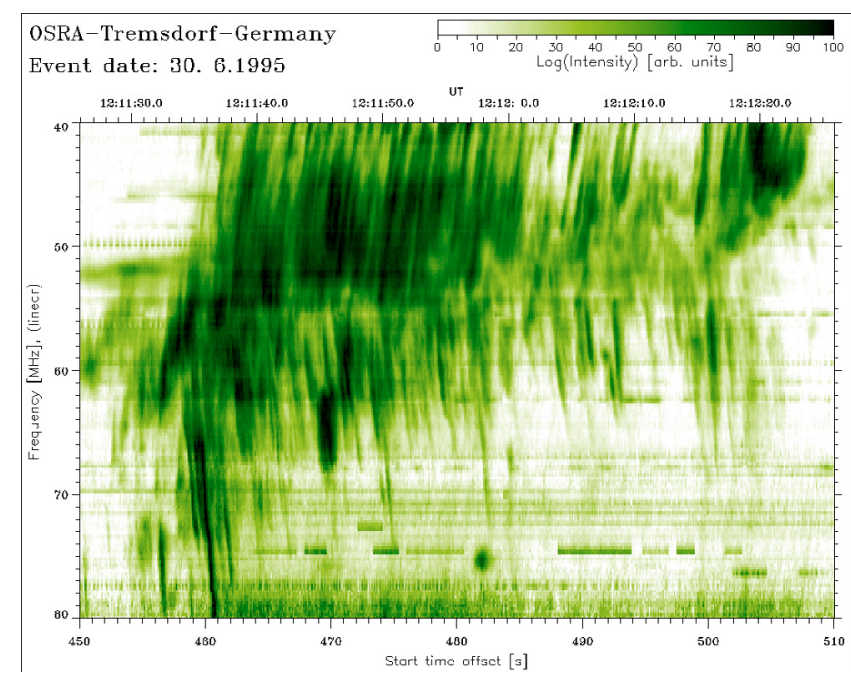

Fig. 1. Dynamic radio spectrum recorded by the radio spectralpolarimeter (Mann et al. 1992) of the Astrophysical Institute Potsdam. It shows an example of a solar type II radio burst. Further explanations are given in Sect. 1.

drift rate of about $-0.1375 \mathrm{MHz} \mathrm{s}^{-1}$ at $55 \mathrm{MHz}$ (whereas Mann \& Klassen (2005) reported mean value on the order of $-0.133 \mathrm{MHz} \mathrm{s}^{-1}$ ), and shows often a fundamental-harmonic structure. It is widely accepted to be the radio signature of a shock wave traveling through the corona (Nelson \& Melrose 1985; Mann 1995; Aurass 1997; Mann 2006). Sometimes, very fast, drifting patches of emission (see Fig. 1) can be seen to emanate from the backbone, toward both lower or higher 
frequencies with typical drift rates of about $\pm 7.2 \mathrm{MHz} \mathrm{s}{ }^{-1}$ at $55 \mathrm{MHz}$ (whereas Mann \& Klassen 2005, reported $\pm 9.1 \mathrm{MHz} \mathrm{s}^{-1}$ ). These features are the so-called herringbone emission, regarded as the radio signature of shock-accelerated electrons (Cairns \& Robinson 1987; Zlobec et al. 1993; Mann \& Klassen 2005).

In the solar corona, the radio emission mechanism is mainly due to plasma emission at the local electron plasma frequency $\omega_{\mathrm{pe}}=\left(4 \pi e^{2} N_{\mathrm{e}} / m_{\mathrm{e}}\right)^{1 / 2}\left(e\right.$, elementary charge; $N_{\mathrm{e}}$, electron number density, $m_{\mathrm{e}}$, electron mass) and/or its harmonics (Melrose $1985)$. Because $\omega_{\text {pe }}$ depends only on the electron number density, the observed frequency corresponds to a certain, local electron number density level. From observations (Newkirk 1961; Koutchmy 1994), one could estimate how the electron number density varies with the distance from the solar surface, so the correspondence of the observed frequency to a certain height of the corona can be established. Thus, a dynamic radio spectrum represents a height-time diagram of the radio source in the solar corona, where high/low frequencies are emitted in the low/high corona due to the gravitational stratification of the solar atmosphere, respectively. According to this, the drift rate $D_{f}$ (i.e., the ratio of the frequency bandwidth $\Delta f$ to the corresponding time duration $\Delta t$ ) at frequency $f$ is related to the radial source velocity $V_{\text {source, } r}$ by

$D_{f}=\frac{f}{2} \frac{1}{N_{\mathrm{e}}} \frac{\mathrm{d} N_{\mathrm{e}}}{\mathrm{d} r} V_{\text {source, } r}$.

Here, $\mathrm{d} N_{\mathrm{e}} / \mathrm{d} r$ denotes the radial density gradient. In the present work, a coronal density model proposed by Newkirk (1961),

$N_{\mathrm{e}}(r)=\alpha N_{0} 10^{4.32\left(R_{\odot} / r\right)}$,

$\left(N_{0}=4.2 \times 10^{4} \mathrm{~cm}^{-3}, R_{\odot}\right.$, radius of the Sun) is used. It corresponds to a barometric density model with a temperature of 1.4 MK (Mann et al. 1999) and agrees very well with the white light scattering measurements by Koutchmy (1994). The coefficient $\alpha$ stands for a density enhancement in the solar corona, where $\alpha=1,4$ should be adopted for quiet equatorial regions and dense coronal loops (Koutchmy 1994), respectively. In the present paper, a Newkirk (1961) model with $\alpha=2$ is used to estimate the plasma parameters in the source region of type II radio bursts, e.g., at $55 \mathrm{MHz}$. Then, the $55 \mathrm{MHz}$ level with an electron number density of $3.75 \times 10^{7} \mathrm{~cm}^{-3}$ is located at a distance of $1.63 R_{\odot}$ from the center of the Sun, i.e., at a height of $440 \mathrm{Mm}$ above the photosphere. There, a magnetic field of $1 \mathrm{G}$ is expected according to Dulk \& McLean (1978), leading to a plasma beta $\beta=8 \pi N k_{\mathrm{B}} T / B^{2}=0.36$ with $N=2 N_{\mathrm{e}}$ as the total particle number density, and additionally, $k_{\mathrm{B}}$ is the Boltzmann's constant, $T$ the temperature, and $B$ the magnetic field. The $55 \mathrm{MHz}$-level provides for the electron cyclotron frequency $\omega_{\text {ce }}=e B /\left(m_{\mathrm{e}} c\right)=1.758 \times 10^{7} \mathrm{~s}^{-1}(c$, velocity of light $)$, for the ratio $\omega_{\mathrm{pe}} / \omega_{\mathrm{ce}}=20$ (with $\omega_{\mathrm{pe}}=2 \pi \times 55 \mathrm{MHz}$ ), and for the Alfvén speed $v_{\mathrm{A}}=360 \mathrm{~km} \mathrm{~s}^{-1}$. Assuming the same density model (Newkirk 1961), the velocities related to the backbone $V_{\mathrm{BB}}$ and herringbone emission $V_{\mathrm{HB}}$ are estimated to be $1000 \mathrm{~km} \mathrm{~s}^{-1}$ and $50000 \mathrm{~km} \mathrm{~s}^{-1}(=0.17 c$, corresponding to a kinetic energy of $7.3 \mathrm{keV}$ ), respectively. Since the backbone is associated with the shock traveling through the corona, coronal shock waves have typical Alfvén Mach numbers $\left(M_{\mathrm{A}}=V_{\mathrm{BB}} / v_{\mathrm{A}}\right)$ of 2.78 .

Holman \& Pesses (1983) proposed that the electrons associated with the herringbones are generated by shock-drift acceleration. Other studies on this subject show that, this mechanism provides a beam-like distribution function for the accelerated electrons (Mann \& Klassen 2005), which corresponds well to the observed characteristics of the herringbone emission stripes. The beam has a velocity

$V_{\text {beam }}=V_{\text {sh }} \sec \theta_{\mathrm{B}, \mathrm{n}}\left(1+\cos ^{2} \alpha_{\mathrm{lc}}\right)$

with $V_{\text {sh }}$ as the shock speed (Mann \& Klassen 2005), and $\theta_{\mathrm{B}, \mathrm{n}}$ denotes the angle between the upstream magnetic field and the shock normal. The loss-cone angle $\alpha_{\text {lc }}$ is defined by the jump of the magnetic field across the shock; i.e., $\alpha_{\mathrm{lc}}=$ $\arcsin \left[\left(B^{\text {up }} / B^{\text {down }}\right)^{1 / 2}\right]$ with $B^{\text {up }}$ and $B^{\text {down }}$ as the magnetic field in the up- and downstream region, respectively. If we apply the shock-drift acceleration as a mechanism for electron acceleration in the case of herringbone emission, we need to take into account the values from the observed drift rates, i.e., $V_{\text {beam }}=$ $V_{\mathrm{HB}}=50000 \mathrm{~km} \mathrm{~s}^{-1}$ and $V_{\mathrm{sh}}=V_{\mathrm{BB}}=1000 \mathrm{~km} \mathrm{~s}^{-1}$. Assuming for the jump of the magnetic field across the shock $B^{\text {down }} / B^{\text {up }}=$ 2, i.e., $\alpha_{\mathrm{lc}}=45^{\circ}$, Eq. (3) requires $\theta_{\mathrm{B}, \mathrm{n}}=88.28^{\circ}$. This result shows that shock-drift acceleration is an efficient mechanism for electron acceleration under coronal conditions only for nearly perpendicular shocks.

Considering this mechanism for the herringbone emission, a nearly perpendicular shock configuration is therefore required to be present during the whole event. For the example shown in Fig. 1, the type II burst appears for about $1 \mathrm{~min}$; i.e., it would cover a spatial scale in the corona of about $60 \mathrm{Mm}$. Even though the shock-drift acceleration is able to explain the electron acceleration rates, the physical conditions required for this mechanism to work are difficult to sustain, since the shock travels more than $60 \mathrm{Mm}$ and needs to be nearly perpendicular all the time. That is an event with low probability. Thus, there is a need for another mechanism for electron acceleration at coronal shocks, and this new mechanism should avoid the restriction of a nearly perpendicular shock geometry. This is the purpose of the presented paper.

Spacecraft observation of the Earth's bow shock, interplanetary shocks, and shocks related to co-rotating interaction regions (CIRs) are the only possibility for in-situ measurements of shocks in space plasmas. Observations by the spacecraft Ulysses at the CIR-related shocks reveal that electrons are really accelerated up to $0.1-0.4 \mathrm{MeV}$ at such shocks (see top panel of Fig. 2). CIR related shocks are efficient at accelerating particles when the conditions $M_{\mathrm{A}}>2.5$ and $50^{\circ}<\theta_{\mathrm{B}, \mathrm{n}}<80^{\circ}$ are simultaneously fulfilled by the shock waves (Classen et al. 1998). Such shocks can be regarded as super-critical shocks (Kennel et al. 1985) and are usually associated with whistler waves in the up- and downstream region, immediately in the vicinity of the shock transition (Gurnett et al. 1985; Classen et al. 1999; Mann et al. 2002). In summary, quasi-perpendicular and super-critical shocks with attached whistler waves tend to generate energetic electrons.

The aim of the present study is to look for a new mechanism for electron acceleration at quasi-perpendicular shocks in the solar corona. Motivated by the observations at CIR related shocks, we consider an electron-whistler-shock interaction. Here, it is basically assumed that the same physical processes for the collisionless shocks in space (e.g., at CIRs) are also true for the coronal shock waves. The paper is organized as follows: the new mechanism is given in Sect. 2, where every stage is explained in the corresponding sections: Sect. 2.1 presents the acceleration of the protons at the shock; the whistler wave excitation due to the proton-wave resonant interaction is shown in Sect. 2.2; in the next section, 2.3, the resonant interaction of the incoming electrons with the excited whistlers is followed in detail; and the Sect. 2.4 includes the mirror reflection of the energized electrons 


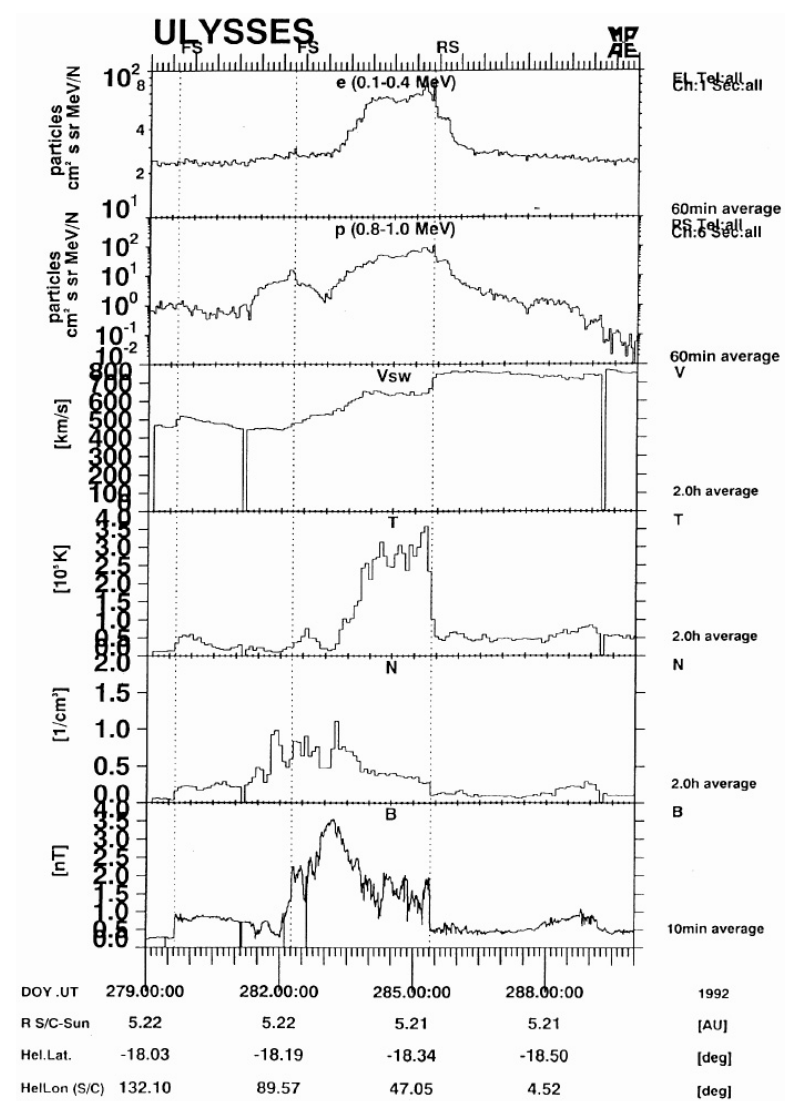

Fig. 2. Behavior of the fluxes of energetic electrons $(0.1-0.4 \mathrm{MeV})$ and protons $(0.8-1.0 \mathrm{MeV})$, the solar wind speed $V_{\mathrm{SW}}$, the proton temperature, the proton number density, and the magnitude of the magnetic field (from top to bottom) during the crossing of a CIR by Ulysses (Scholer et al. 1999).

at the shock that propagate in the upstream region later on. The results are discussed in Sect. 3. In addition, the description of the whistler wave fields and the general theory on the electron motion in homogeneous magnetic field as used in Sect. 2 are presented in Appendices A and B, correspondingly.

\section{The model}

The model presented in this paper considers the following scenario (schematically shown in Fig. 3): a shock wave is moving through the solar atmosphere with a velocity of about $1000 \mathrm{~km} \mathrm{~s}^{-1}$. The inflowing protons (i.e., toward the shock) can be successfully accelerated at the shock. Due to their relatively slow thermal velocity (on the order of $110 \mathrm{~km} \mathrm{~s}^{-1}$ for a typical coronal temperature of $1.4 \mathrm{MK}$ ), the quasi-perpendicular configuration $\left(\theta_{\mathrm{B}, \mathrm{n}} \approx 70^{\circ}\right)$ is sufficient for a substantial velocity gain for the protons (of about $6000 \mathrm{~km} \mathrm{~s}^{-1}$ ). The protons are accelerated and reflected at the shock back to the upstream region, where they are able to excite whistler waves by resonant waveparticle interaction. These excited whistlers can only interact with the inflowing electrons, which fulfill the resonance condition. These electrons are accelerated (i.e., gain energy) in the whistler electric field. Consequently, the perpendicular electron velocity component with respect to the ambient magnetic field is increasing during this resonant interaction. That also leads to an increase in its magnetic moment. Simultaneously with this process, the accelerating electrons are moving toward the shock front. Since the shock is accompanied by a jump in the magnetic

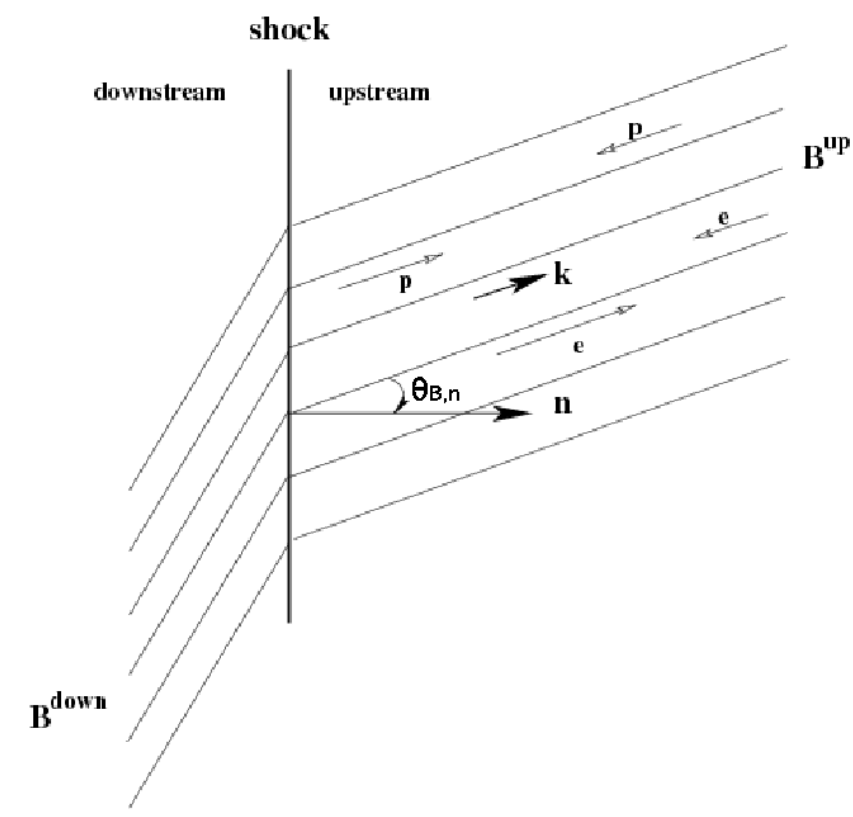

Fig. 3. Sketch of the shock configuration. Both the in-flowing toward the shock front and out-flowing protons ("p") and electrons ("e") move along the upstream magnetic field lines $\left(\boldsymbol{B}^{\text {up }}\right)$. The shock moves along the shock normal $(\boldsymbol{n})$. The excited whistler waves (denoted with their wave vector $\boldsymbol{k}$ ) propagate away from the shock along the magnetic field lines, which is chosen to be the positive direction.

field, it represents a traveling magnetic mirror. Finally, the electrons are mirror reflected at the shock, and they propagate into the upstream region. The outflowing electrons (i.e., away from the shock) do not fulfill the resonance condition so are not in resonance with the whistler wave. Then, they can travel undisturbed into the far upstream region and be detected as herringbone emission, for example.

\subsection{Inflow of the upstream protons and their acceleration}

It is well-known from observations at Earth's bow shock (Paschmann et al. 1980, 1981) that protons are reflected and accelerated by nearly conserving their magnetic moment at quasiperpendicular, super-critical shocks. This process occurs in the range $30^{\circ}<\theta_{\mathrm{B}, \mathrm{n}}<75^{\circ}$, with very few events observed for $\theta_{\mathrm{B}, \mathrm{n}}>75^{\circ}$, as reported by Paschmann et al. (1981, see also Fig. 3 there). The reflected protons establish a beam-like distribution with a strong temperature anisotropy. The beam density is typically in the range $0.3 \%$ to $13 \%$ of the number density of the incoming protons (Paschmann et al. 1981). Since, under coronal conditions, a quasi-perpendicular shock with an Alfvén Mach number $M_{\mathrm{A}}=2.76$ is super-critical (Kennel et al. 1985; Mann et al. 1995), it is assumed that protons are also reflected and accelerated at coronal shock waves. The proton (denoted with a superscript "i") velocity gain parallel to the upstream magnetic field can be expressed by

$V_{\|, \text {ref }}^{\mathrm{i}}=2 V_{\mathrm{sh}} \sec \theta_{\mathrm{B}, \mathrm{n}}-V_{\|, \text {in }}^{\mathrm{i}}$

(Paschmann et al. 1980; Holman \& Pesses 1983), where the subscript "in" denotes the initial velocity of the particle and "ref" the reflected one. For the protons in the initial state, a Maxwellian distribution normalized to unity is assumed, namely

$f_{\mathrm{i}}(V)=\frac{1}{\left(2 \pi V_{\mathrm{th}, \mathrm{i}}^{2}\right)^{3 / 2}} \mathrm{e}^{-V^{2} /\left(2 V_{\mathrm{th}, \mathrm{i}}^{2}\right)}$ 
with $V_{\text {th,i }}=\left(k_{\mathrm{B}} T / m_{\mathrm{i}}\right)^{1 / 2}\left(m_{\mathrm{i}}\right.$, proton mass $)$ as the thermal proton velocity. Then, the number of particles with velocities parallel to the ambient magnetic field in the range $-V_{u}<V_{\|}<V_{u}$ is given by

$$
\begin{aligned}
\frac{N\left(V_{u}\right)}{N_{0}} & =\frac{2 \pi}{\left(2 \pi V_{\mathrm{th}, \mathrm{i}}^{2}\right)^{3 / 2}} \int_{-V_{u}}^{V_{u}} \mathrm{~d} V_{\|} \int_{0}^{\infty} \mathrm{d} V_{\perp} V_{\perp} \mathrm{e}^{-\frac{V_{\|}^{2}+V_{\perp}^{2}}{2 V_{\mathrm{th}, \mathrm{i}}^{2}}} \\
& =\int_{-V_{u}}^{V_{u}} \mathrm{~d} V_{\|} F\left(V_{\|}\right) \\
& =2 \Phi\left(V_{u} / V_{\mathrm{th}, \mathrm{i}}\right)
\end{aligned}
$$

with $N_{0}$ as the total electron number density and

$\Phi\left(V_{u} / V_{\mathrm{th}, \mathrm{i}}\right)=\frac{1}{(2 \pi)^{1 / 2}} \int_{0}^{V_{u} / V_{\mathrm{th}, \mathrm{i}}} \mathrm{d} t \mathrm{e}^{-t^{2} / 2}$.

In Eq. (6), $F\left(V_{\|}\right)$denotes the reduced distribution function resulting after the integration with respect to the perpendicular velocity component, i.e.,

$F\left(V_{\|}\right)=\frac{1}{\left(2 \pi V_{\mathrm{th}, \mathrm{i}}^{2}\right)^{1 / 2}} \mathrm{e}^{-V_{\|}^{2} /\left(2 V_{\mathrm{th}, \mathrm{i}}^{2}\right)}$.

Equation (6) provides $99 \%$ of all particles having velocities parallel to the ambient magnetic field in the range $-2.576 V_{\mathrm{th}, \mathrm{i}}<$ $V_{\|, \text {in }}^{\mathrm{i}}<2.576 V_{\mathrm{th}, \mathrm{i}}$. For a coronal temperature of $T=1.4 \mathrm{MK}$, the thermal velocity of the protons is obtained to be $V_{\mathrm{th}, \mathrm{i}}=$ $107.5 \mathrm{~km} \mathrm{~s}^{-1}$. That value is essentially lower than the shock speed of $1000 \mathrm{~km} \mathrm{~s}^{-1}$. Taking into account that the initial proton velocities parallel to the ambient magnetic field are in the range given above, i.e. $-277 \mathrm{~km} \mathrm{~s}^{-1}<V_{\| \text {,in }}^{\mathrm{i}}<277 \mathrm{~km} \mathrm{~s}^{-1}$, and substituting the values $V_{\mathrm{sh}}=1000 \mathrm{~km} \mathrm{~s}^{-1}$ and $\theta_{\mathrm{B}, \mathrm{n}}=70^{\circ}$ in Eq. (4), one obtains the final speed of the reflected protons in the range $5570<V_{\|, \text {ref }}^{\mathrm{i}}<6130 \mathrm{~km} \mathrm{~s}^{-1}$ (or $0.0186<\beta_{\|}^{\mathrm{i}}=$ $\left.V_{\|, \text {ref }}^{\mathrm{i}} / c<0.0204\right)$. For smaller angles, e.g., $\theta_{\mathrm{B}, \mathrm{n}}=55^{\circ}$, the resulting final speed of protons shifts to lower velocities, e.g., to $3210<V_{\|, \text {ref }}^{\mathrm{i}}<3760 \mathrm{~km} \mathrm{~s}^{-1}$ (or $0.0107<\beta_{\|}^{\mathrm{i}}=V_{\|, \text {,ef }}^{\mathrm{i}} / c<$ $0.0125)$. Further on, an angle of $\theta_{\mathrm{B}, \mathrm{n}}=70^{\circ}$ is used for discussion, since it can be considered as a representative value for quasiperpendicular shocks. Here, the shock propagation is chosen in the positive direction, and the reflected and accelerated protons are moving in the same direction, i.e., away from the shock.

That demonstrates that the protons get a substantial gain in energy due to the encounter with the quasi-perpendicular coronal shock wave.

\subsection{Whistler excitation}

To excite whistler waves by wave-particle interaction in the case of the accelerated protons, they need to fulfill the resonance condition; see Baumjohann \& Treumann (1997),

$k c \beta_{\|}^{\mathrm{i}}-\omega-\omega_{\mathrm{ci}}=0$,

where $k, \omega$, and $\omega_{\mathrm{ci}}=e B /\left(m_{\mathrm{i}} c\right)$ are the wave number, the wave frequency, and the proton cyclotron frequency, respectively. Because whistler waves are predominantly excited along the magnetic field (Vocks \& Mann 2006), circularly polarized waves propagating along to the ambient magnetic field lines are only considered here. Their index of refraction is given by (Baumjohann \& Treumann 1997),

$$
\frac{k^{2} c^{2}}{\omega^{2}}=1-\frac{\omega_{\mathrm{pe}}^{2}}{\omega\left(\omega \pm \omega_{\mathrm{ce}}\right)}-\frac{\omega_{\mathrm{pi}}^{2}}{\omega\left(\omega \mp \omega_{\mathrm{ci}}\right)} \text {. }
$$

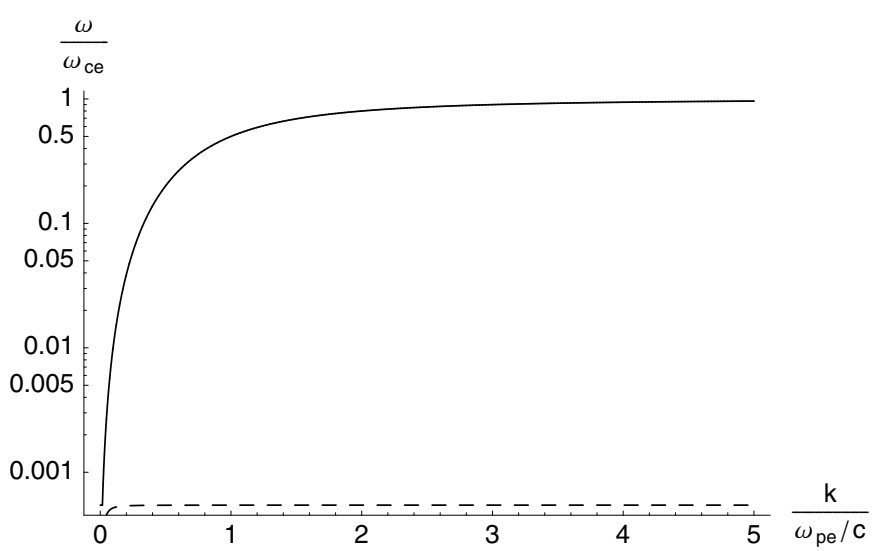

Fig. 4. Dispersion relation for the LCP (dashed line) Alfvén and RCP (solid line) whistler wave for $S=20$. Both propagate along the ambient magnetic field.

Here, the upper and lower signs stand for left- (LCP) and righthand (RCP) circular polarization, respectively. The corresponding dispersion relations are depicted for $S=\omega_{\text {pe }} / \omega_{\text {ce }}=20$ in Fig. 4. At this point, the following normalization is introduced for the wave number $k^{\prime}=k c / \omega_{\text {pe }}$ and the frequency $\omega^{\prime}=\omega / \omega_{\text {ce }}$, correspondingly. Here, the primed quantities are already dimensionless. Substituting these expressions back into the Eqs. (9) and (10), we obtain the normalized ion resonance condition and the RCP wave dispersion relation, respectively,

$S k^{\prime} \beta_{\|}^{\mathrm{i}}-\omega^{\prime}-\mu_{\mathrm{e}}=0$

$\frac{k^{\prime 2}}{\omega^{\prime 2}}=\frac{1}{S^{2}}+\frac{1}{\omega^{\prime}-\omega^{\prime 2}}$,

in the case of $\omega^{\prime} \gg \mu_{\mathrm{e}}\left(\mu_{\mathrm{e}}=m_{\mathrm{e}} / m_{\mathrm{i}}\right.$, electron-to-proton mass ratio). In the case of coronal plasma at the $55 \mathrm{MHz}-$ level, the frequency ratio $S$ is estimated as $S=20$ (see Sect. 1). Hence, neglecting the $S^{-2}$ factor in Eq. (12), the normalized dispersion relation for the whistler waves is found to be

$k^{\prime}=\sqrt{\frac{\omega^{\prime}}{1-\omega^{\prime}}}$.

This relation is substituted into the normalized ion resonance condition (11), leading to

$\beta_{\|}^{\mathrm{i}}=\frac{1}{S} \sqrt{\omega^{\prime}\left(1-\omega^{\prime}\right)}$

In order to obtain an estimation for the frequencies of the excited whistlers, $0.0186<\beta_{\|}^{\mathrm{i}}<0.0204$ (see Sect. 2.1) are employed for $\beta_{\|}^{\mathrm{i}}$ in Eq. (14) resulting in $0.166<\omega^{\prime}<0.211$ (or equivalently from Eq. (13), $\left.0.446<k^{\prime}<0.517\right)$. In other words, the shockaccelerated protons excite a spectrum of whistler waves predominantly enhanced in the frequency range $0.166<\omega^{\prime}<0.211$ with a maximum at $\omega^{\prime}=0.188$ (and $k^{\prime}=0.4815$ ). In the case of an initial Maxwellian velocity distribution function, see Eq. (5), most of the in-flowing protons have a velocity $V_{\|, \text {in }}^{\mathrm{i}}=0$ parallel to the magnetic field. After the reflection at the shock, they have a velocity of $5850 \mathrm{~km} \mathrm{~s}^{-1}$ (or $\left.\beta_{\|}^{\mathrm{i}}=0.0195\right)$ and are able to excite whistlers with $\omega^{\prime}=0.188$ according Eq. (14). Therefore, the final whistler spectrum should have a maximum at $\omega^{\prime}=0.188$.

From the resonance condition Eq. (9), one gets $\omega_{\mathrm{ci}}=$ $k\left(V_{\|}^{\mathrm{i}}-V_{\mathrm{ph}}\right)$ with the whistler-phase velocity $V_{\mathrm{ph}}=\omega / k$. Since 
the proton cyclotron frequency $\omega_{\mathrm{ci}}$ is positive, the proton velocity must be faster than the phase speed of the whistlers, so that the righthand, circularly polarized whistlers appear to be lefthanded in the rest frame of the energetic protons. Thus, the shock accelerated protons do fulfill both the resonance condition and the condition of providing the right whistler polarization, which is lefthand circularly polarized in the proton rest frame, but righthand circularly polarized in the laboratory frame.

Simultaneously, the shock accelerated protons can also interact with low-frequency lefthand circularly polarized Alfvén waves according to the resonance condition (Baumjohann \& Treumann 1997),

$k c \beta_{\|}^{\mathrm{i}}-\omega+\omega_{\mathrm{ci}}=0$.

Employing the normalization introduced above, the normalized resonance condition and the wave dispersion relation are given by

$S k^{\prime} \beta_{\|}^{\mathrm{i}}-\omega^{\prime}+\mu_{\mathrm{e}}=0$

and

$k^{\prime}=\sqrt{\frac{\left(1+\mu_{\mathrm{e}}\right) \omega^{\prime 2}}{\left(1+\omega^{\prime}\right)\left(\mu_{\mathrm{e}}-\omega^{\prime}\right)}}$.

Then, the accelerated protons in the range $0.0186<\beta_{\|}^{\mathrm{i}}<0.0204$ excite Alfvén waves in the frequency range $2.87 \times 10^{-5}<\omega^{\prime}<$ $3.13 \times 10^{-5}$ or $(0.053-0.057) \omega_{\text {ci }}$. But these Alfvén waves are propagating in the opposite direction to the protons, i.e., towards the shock, as results from the inspection of Eq. (15).

In summary, shock-accelerated protons are able to excite both whistler waves with frequencies in the range $(0.166-0.211) \omega_{\text {ce }}$ and low-frequency Alfvén waves. These whistlers propagate away from the shock in the upstream region, whereas the Alfvén waves travel towards the shock (Winske \& Leroy 1984).

\subsection{Resonant electron-whistler interaction}

The excited whistler waves can resonantly interact with the electrons in the upstream region. The electron resonance conditions is given by

$k c \beta_{\|}^{\mathrm{e}}-\omega+\omega_{\mathrm{ce}}=0$,

with $\beta_{\|}^{\mathrm{e}}=V_{\|}^{\mathrm{e}} / c$; see Baumjohann \& Treumann (1997). The electron part is denoted with superscript "e". Then, one gets

$S k^{\prime} \beta_{\|}^{\mathrm{e}}-\omega^{\prime}+1=0$

after the usual normalization (see Sect. 2.2). Using relation (13) again for the normalized wave number, the equation above becomes

$-\beta_{\|}^{\mathrm{e}}=\frac{1}{S} \sqrt{\frac{\left(1-\omega^{\prime}\right)^{3}}{\omega^{\prime}}}$.

Thus, only counter-streaming (i.e., moving towards the shock) electrons are in resonance with the whistler waves. Since a whistler wave spectrum covering the frequency range $0.166<$ $\omega^{\prime}<0.211$ is present in the upstream region, they can be in resonance with electrons in the velocity range $-0.093<\beta_{\|}^{\text {e }}<$ -0.076 , if one adopts $S=20$.

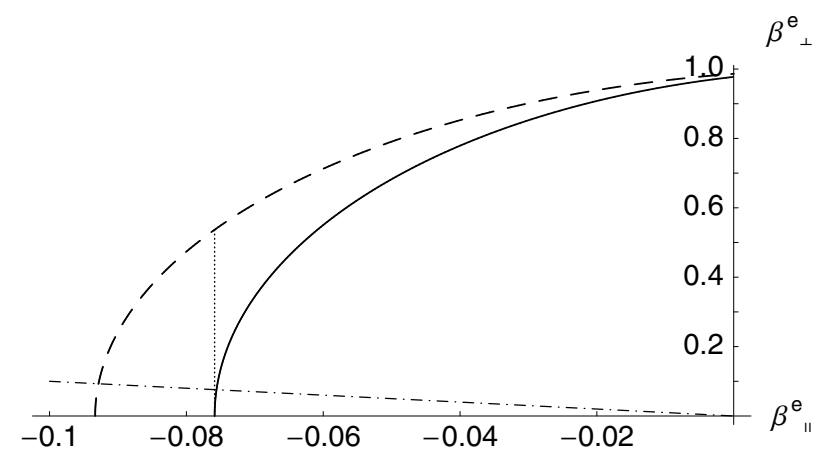

Fig. 5. Solution of the resonance curve, Eq. (22), for $S=20$ and $\omega^{\prime}=$ 0.166 (dashed) and 0.211 (solid line) in the $\left(\beta_{\|}^{\mathrm{e}}, \beta_{\perp}^{\mathrm{e}}\right)$-plane. The dasheddotted line corresponds to a pitch angle of $45^{\circ}$.

To study relativistic electrons, one needs to correct the above result using the extended, relativistic resonance condition; i.e.,

$k c \beta_{\|}^{\mathrm{e}}-\omega+\omega_{\text {ce }}\left[1-\left(\beta_{\|}^{\mathrm{e}}\right)^{2}-\left(\beta_{\perp}^{\mathrm{e}}\right)^{2}\right]^{1 / 2}=0$,

see Baumjohann \& Treumann (1997). Employing the same normalization, the above equation becomes

$S k^{\prime} \beta_{\|}^{\mathrm{e}}-\omega^{\prime}+\left[1-\left(\beta_{\|}^{\mathrm{e}}\right)^{2}-\left(\beta_{\perp}^{\mathrm{e}}\right)^{2}\right]^{1 / 2}=0$.

Taking relation (13) into account, an expression for the parallel and the perpendicular component of the velocity is obtained from Eq. (22) that is plotted in Fig. 5 in the $\left(\beta_{\|}^{\mathrm{e}}, \beta_{\perp}^{\mathrm{e}}\right)$-plane. There, one could see two curves, corresponding to the minimum (0.076) and maximum (0.093) values of $\left|\beta_{\|}^{\mathrm{e}}\right|$ at $\beta_{\perp}^{\mathrm{e}}=0$. These two curves define the area in the $\left(\beta_{\|}^{\mathrm{e}}, \beta_{\perp}^{\mathrm{e}}\right)$-plane that is usually called "resonance band". Only electrons that are initially located in this "resonance band" are able to resonantly interact with the whistlers. The appearance of such an area is immediately related to the thermal spread of the inflowing protons and, consequently, with the excitation of a whistler spectrum in a finite frequency range. The curvature (as enhanced on the plot) is due to the relativistic effects, since in the nonrelativistic case it would be two straight lines parallel to the $\beta_{\perp}^{\mathrm{e}}$-axis. During the resonant interaction with the whistler wave field, the electron is increasing its velocity component perpendicular to the magnetic field. That represents a motion upward in the $\left(\beta_{\|}^{\mathrm{e}}, \beta_{\perp}^{\mathrm{e}}\right)$-plane (see Fig. 5) between these curves or in the so-called "resonance band", leading to an increase in its kinetic energy (see Fig. 6). Normalized to the rest energy $\left(m_{\mathrm{e}} c^{2}=512 \mathrm{keV}\right)$, the kinetic energy of the electron is usually defined by

$\frac{W_{\text {kin }}}{m_{\mathrm{e}} c^{2}}=\frac{1}{\sqrt{1-\beta^{2}}}-1$,

with $\beta^{2}=\left(\beta_{\|}^{\mathrm{e}}\right)^{2}+\left(\beta_{\perp}^{\mathrm{e}}\right)^{2}$. When the relation (22) is taken into account, the kinetic energy (23) can be plotted in terms of the perpendicular velocity component $\beta_{\perp}^{\text {e }}$ (see Fig. 6). A substantial energy gain for the electrons can be noticed due to the resonant electron-whistler interaction.

In general, the movement of an electron in given electric and magnetic fields can be described by the momentum

$\frac{\mathrm{d} \boldsymbol{p}}{\mathrm{d} t}=-e[\boldsymbol{E}+\boldsymbol{\beta} \times \boldsymbol{B}]$,

and the kinematic equation (Baumjohann \& Treumann 1997)

$\frac{\mathrm{d} \boldsymbol{r}}{\mathrm{d} t}=c \boldsymbol{\beta}$, 


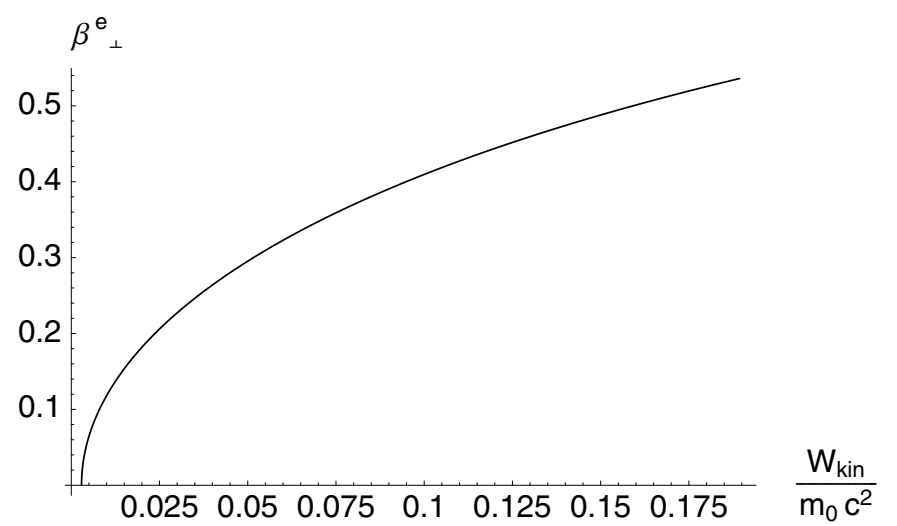

Fig. 6. Relation between $\beta_{\perp}^{\mathrm{e}}$ and the kinetic energy $W_{\text {kin }} / m_{\mathrm{e}} c^{2}$, Eq. (23), where $\left|\beta_{\|}^{\mathrm{e}}\right|=0.076$ and $\beta_{\perp}^{\mathrm{e}}$ takes the values along the dotted line in Fig. 5.

with $\boldsymbol{\beta}=\boldsymbol{v} / c$. The momentum is defined by $\boldsymbol{p}=m_{0} c \boldsymbol{\beta} / \sqrt{1-\beta^{2}}$. To normalize the above equations of motion, the following dimensionless quantities are introduced: time, $t^{\prime}=t \omega_{\mathrm{ce}}$; spatial coordinate, $r^{\prime}=r \omega_{\text {pe }} / c$; momentum, $p^{\prime}=p /\left(m_{0} c\right)$; energy, $W_{\text {kin }}^{\prime}=W_{\text {kin }} /\left(m_{0} c^{2}\right)$; the magnetic and electric fields are normalized to the background (upstream) magnetic field, $B_{0}$, and $B_{0} / S\left(S=\omega_{\mathrm{pe}} / \omega_{\mathrm{ce}}\right)$, respectively. Substituting these normalizations into the above equations of motion, one gets the following normalized system of equations,

$$
\begin{aligned}
& \frac{\mathrm{d} \boldsymbol{p}^{\prime}}{\mathrm{d} t^{\prime}}=-\boldsymbol{E}^{\prime}-\boldsymbol{\beta} \times \boldsymbol{B}^{\prime}, \\
& \frac{\mathrm{d} \boldsymbol{r}^{\prime}}{\mathrm{d} t^{\prime}}=S \boldsymbol{\beta} .
\end{aligned}
$$

Note that Eq. (23) is already normalized. In order to find the energy gain, the time derivative of Eq. (23) is calculated, i.e.,

$\dot{W}_{\text {kin }}^{\prime}=\frac{\beta \cdot \dot{\beta}}{\left(1-\beta^{2}\right)^{3 / 2}}$,

which gives the same result as a dot-product of Eq. (26) with $\boldsymbol{\beta}$ (note that all betas here are vector quantities). Finally using the relation $\beta=\boldsymbol{p}^{\prime} /\left(1+p^{\prime 2}\right)^{1 / 2}$, one obtains the following expression for the time derivative of the normalized kinetic energy,

$\dot{W}_{\text {kin }}^{\prime}=-\frac{\boldsymbol{E}^{\prime} \cdot \boldsymbol{p}^{\prime}}{\left(1+p^{\prime 2}\right)^{1 / 2}}$.

Thus, the energy gain can be obtained if one knows the wave electric field and the momentum of the particle. Substituting in the above expression both the electric field from the whistler wave field and the momentum from the electron motion in a homogeneous magnetic field (see Appendices A and B), one obtains the following result for the energy gain:

$\dot{W}_{\text {kin }}^{\prime}=\frac{V_{\mathrm{ph}}^{\prime} \beta_{\perp}^{\mathrm{e}} B_{\mathrm{wh}}^{\prime}}{S} \sin \left(\alpha_{1}+\alpha_{2}\right)$

with $V_{\mathrm{ph}}^{\prime}=\omega^{\prime} / k^{\prime}, \alpha_{1}=k^{\prime} x^{\prime}\left(t^{\prime}\right)-\omega^{\prime} t^{\prime}, \alpha_{2}=\omega_{\mathrm{ce}}^{\prime} t^{\prime}+\phi_{0}$. The normalized frequency and the amplitude of the whistler magnetic field (i.e., as a fraction of the upstream one) are $\omega^{\prime}$ and $B_{\text {wh }}^{\prime}$, respectively. Introducing the solution for $x^{\prime}\left(t^{\prime}\right)$ (see Eq. (B.10) in Appendix B), the argument of the sin-function, $\alpha_{1}+\alpha_{2}$, can be expressed by

$\alpha_{1}+\alpha_{2}=D t^{\prime}+\varphi_{0}$ where

$$
\begin{aligned}
& D=k^{\prime} S \beta_{\|}^{\mathrm{e}}-\omega^{\prime}+\left(1+p^{\prime 2}\right)^{-1 / 2}, \\
& \varphi_{0}=k^{\prime} x_{0}+\phi_{0} .
\end{aligned}
$$

Here, $x_{0}$ and $\phi_{0}$ are the initial conditions for the spatial coordinate and the phase, respectively. The temporal integration of Eq. (30) leads to

$\Delta W_{\mathrm{kin}}^{\prime}=-\frac{V_{\mathrm{ph}}^{\prime} \beta_{\perp}^{\mathrm{e}} B_{\mathrm{wh}}^{\prime}}{D S}\left[\cos \left(D t^{\prime}+\varphi_{0}\right)-\cos \varphi_{0}\right]$,

which represents a temporally oscillating function. $\Delta W_{\text {kin }}^{\prime}$ only increases with time, if the condition $D \rightarrow 0$ is fulfilled (exactly the resonance condition (22)), resulting in

$\Delta W_{\mathrm{kin}}^{\prime}=\frac{V_{\mathrm{ph}}^{\prime} \beta_{\perp}^{\mathrm{e}} B_{\mathrm{wh}}^{\prime}}{S} t^{\prime} \sin \varphi_{0}$.

That demonstrates analytically that the resonant electronwhistler interaction really leads to a gain in energy. From Eq. (34), it follows that the particles either gain or lose energy due to the resonant interaction, if phase $\varphi_{0}$ is in the range $0<\varphi_{0}<\pi$ and $\pi<\varphi_{0}<2 \pi$, respectively. Assuming that the inflowing electrons are gyrotropically distributed, one half of them are accelerated, whereas the other half is decelerated with respect to the velocity perpendicular to the ambient magnetic field. Averaging over all angles $\varphi_{0}$ in the range $0<\varphi_{0}<\pi$, i.e.,

$\frac{1}{\pi} \int_{0}^{\pi} \sin \varphi_{0} \mathrm{~d} \varphi_{0}=\frac{2}{\pi}$

one gets for the mean temporal energy gain of the accelerated electrons

$\Delta \bar{W}_{\mathrm{kin}}^{\prime}=\frac{2 V_{\mathrm{ph}}^{\prime} \beta_{\perp}^{\mathrm{e}} B_{\mathrm{wh}}^{\prime}}{\pi S} t^{\prime}$.

As already mentioned, the gain and the loss of energy happens due to the change of the particle velocity perpendicular to the ambient magnetic field because of the resonant electron-whistler interaction. Since a Maxwellian distribution, which is a monotonically decreasing function with respect to $V_{\perp}$, is assumed in the initial state, see Eqs. (5) and (6), there are more particles that are accelerated than those that are decelerated. That finally leads to a net energy gain for the whole population located in the "resonance band" in the $\left(\beta_{\|}^{\mathrm{e}}, \beta_{\perp}^{\mathrm{e}}\right)$-plane.

\subsection{Magnetic mirroring at the shock}

A fast magnetosonic shock wave is accompanied by a jump in the density and the magnetic field. In this sense, it represents a moving magnetic mirror on which charged particles can be reflected and subsequently accelerated. In a weakly varying magnetic field, i.e., $\left|r_{\mathrm{L}} \nabla B / B\right| \ll 1$, where $r_{\mathrm{L}}$ is the Larmor radius, the particle motion is governed by the conservation of the kinetic energy (or amount of momentum) and the magnetic moment (Baumjohann \& Treumann 1997), i.e.,

$p_{\|, j}^{2}+p_{\perp, j}^{2}=p_{\|, 0}^{2}+p_{\perp, 0}^{2}$

$\frac{p_{\perp}^{2}}{B_{j}}=\frac{p_{\perp, 0}^{2}}{B_{0}}$ 
where the index " 0 " denotes the values of the corresponding quantities in the undisturbed (i.e., upstream) region and the index " $j$ " denotes any subsequent temporal states. One can combine the above equations into the following expression

$$
\frac{p_{\|, j}^{2}}{p_{\|, 0}^{2}}=1-\left(\frac{B_{j}}{B_{0}}-1\right) \frac{p_{\perp, 0}^{2}}{p_{\|, 0}^{2}} .
$$

Now, two important angles, as commonly used, are also introduced here: the pitch angle,

$\alpha_{j}=\arctan \frac{p_{\perp, j}}{p_{\|, j}}$,

and the loss-cone angle,

$\alpha_{\mathrm{lc}, j}=\arcsin \sqrt{\frac{B_{0}}{B_{j}}}$,

and hence Eq. (39) can be rewritten as

$$
\frac{p_{\|, j}^{2}}{p_{\|, 0}^{2}}=1-\frac{\tan ^{2} \alpha_{0}}{\tan ^{2} \alpha_{\mathrm{lc}, j}},
$$

where $\alpha_{0}=\arctan \left(p_{\perp, 0} / p_{\|, 0}\right)$ is the initial pitch angle of the particles and depends on the initial conditions observed in the upstream region. The inspection of Eq. (39) leads to all particles with an initial pitch angle $\alpha_{0}>\alpha_{\mathrm{lc}, j}$ being reflected. Identifying $B_{0}$ and $B_{j}$ with the magnetic fields $B^{\text {up }}$ and $B^{\text {down }}$ in the upand downstream region, respectively, all particles with an initial pitch angle

$\alpha_{0}>\alpha_{\mathrm{lc}, \mathrm{sh}}=\arcsin \left(\frac{B^{\mathrm{up}}}{B^{\text {down }}}\right)^{1 / 2}$

will be reflected by their encounter with the shock. The other ones are transmitted into the downstream region.

Since the shock is moving, the consideration presented above must actually be made in the so-called de Hoffmann-Teller frame; see e.g., Mann \& Klassen (2005). The shock speed in the de Hoffmann-Teller frame is given by $V_{\mathrm{sh}}^{\mathrm{HT}}=V_{\mathrm{sh}} \sec \theta_{\mathrm{B}, \mathrm{n}}$ (Mann $\&$ Klassen 2005), which has a typical value of $3000 \mathrm{~km} \mathrm{~s}^{-1}$ adopting $V_{\mathrm{sh}}=1000 \mathrm{~km} \mathrm{~s}^{-1}$ and $\theta_{\mathrm{B}, \mathrm{n}}=70^{\circ}$. Since this value is essentially lower than the thermal electron velocity $V_{\text {th,e }}=$ $\left(k_{\mathrm{B}} T / m_{\mathrm{e}}\right)^{1 / 2}$, which has a typical value of $4600 \mathrm{~km} \mathrm{~s}^{-1}$ for a coronal temperature of $1.4 \mathrm{MK}$, the influence of the motion of the shock on the electron reflection process can be neglected for considering electrons that has been done in this section. Due to the motion of the shock, the electrons reflected back at it get an additional energy gain due to shock drift acceleration, but it can be neglected in the case of quasi-perpendicular shocks under coronal conditions.

As already discussed, the electrons increase their momentum perpendicular to the ambient magnetic field due to the resonant electron-whistler interaction (see Fig. 5) and, consequently, their pitch angle (see Eq. (40)) as well. Thus, a major part of the energized electrons fulfill the reflection condition (43) and, finally, move away from the shock into the upstream region. After the reflection, the accelerated electrons are co-streaming with the whistlers and, thus, do not fulfill the resonance condition (22); i.e., they are out of resonance and can freely reach the far upstream region.

\section{Discussion and summary}

In the framework of the proposed mechanism, the electron acceleration happens in the $\left(\beta_{\perp}^{\mathrm{e}}, \beta_{\|}^{\mathrm{e}}\right)$-plane due to the resonant electron-whistler interaction as illustrated in Fig. 5. It actually represents a motion toward higher values of $\beta_{\perp}^{\mathrm{e}}$ within the socalled "resonance band" $\left(\beta_{\perp}^{\mathrm{e}}, \beta_{\|}^{\mathrm{e}}\right)$-plane. Figure 5 results from parameters, which are appropriate for a shock wave associated with solar type II radio bursts around $55 \mathrm{MHz}$. In the initial state, the electron must be located in the "resonance band" in order to become energized. In the "resonance band", the electrons with the lowest initial energy have values of $\left|\beta_{\|}^{\mathrm{e}}\right|=0.076$ and $\beta_{\perp}^{\mathrm{e}}=0$; i.e., they have an initial energy of $1.5 \mathrm{keV}$. In the case of the most efficient energy gain, they move upward along a straight line (see Fig. 5) parallel to the $\beta_{\perp}^{\mathrm{e}}$-axis. They leave the "resonance band" at the point $\left(\left|\beta_{\|}^{\mathrm{e}}\right|=0.076, \beta_{\perp}^{\mathrm{e}}=0.537\right)$ in the $\left(\beta_{\perp}^{\mathrm{e}}, \beta_{\|}^{\mathrm{e}}\right)$-plane. Then, they have a final kinetic energy of $97 \mathrm{keV}$. During this process, the pitch angle changes from $0^{\circ}$ to $82^{\circ}$. Particles with such a final pitch angle are reflected at shock, since their pitch angle is greater than the loss-cone angle $\alpha_{\mathrm{lc}, \mathrm{sh}}=45^{\circ}$ (in Fig. 5) as required for reflection, see Eq. (43). Such a value is obtained for the loss-cone angle, if one assumes a jump in the magnetic field $B^{\text {down }} / B^{\text {up }}=2$ as a typical value for coronal shock waves.

The inspection of Fig. 5 reveals that only electrons, which are initially located within the "resonance band" in the $\left(\beta_{\perp}^{\mathrm{e}}, \beta_{\|}^{\mathrm{e}}\right)$-plane, are accelerated; i.e., their initial energy must be $\geq 1.5 \mathrm{keV}$ corresponding to a velocity of $0.076 \mathrm{c}=4.96 V_{\mathrm{th}, \mathrm{e}}$ (with $V_{\text {th,e }}=4600 \mathrm{~km} \mathrm{~s}^{-1}$ as the thermal electron speed for a coronal temperature of $1.4 \mathrm{MK}$ ). In the case of an initial Maxwellian distribution of the electrons in the upstream region, the number density of the electrons initially located within the "resonance band" can be estimated to be $\approx 3.582 \times 10^{-7} N_{\mathrm{e}}$. (In this case the integration of the Maxwellian distribution, see Eqs. (7) and (8), is performed from $0.076 c / V_{\text {th,e }}$ to $0.093 c / V_{\text {th,e, }}$, which in this case are 4.96 and 6.06 of the electron thermal speed, correspondingly.) Only these electrons can really be accelerated by the proposed mechanism; i.e., only a minor part of the electrons in the upstream region gain energy.

Now, the energy budget of the different stages of the whole process is discussed here. As already mentioned, those particles that are initially located in the "resonance band" of the $\left(\beta_{\perp}^{\mathrm{e}}, \beta_{\|}^{\mathrm{e}}\right)$-plane are finally accelerated. These are $N_{\mathrm{acc}}=3.582 \times$ $10^{-7} N_{\mathrm{e}}=13.43 \mathrm{~cm}^{-3}$ electrons with $N_{\mathrm{e}}=3.57 \times 10^{7} \mathrm{~cm}^{-3}$. Assuming $\left|\beta_{\|}^{\mathrm{e}}\right|=0.076$ and $\beta_{\perp}^{\mathrm{e}} / 2=0.537 / 2=0.2685$ (see Fig. 5), as mean values of the accelerated electrons, the kinetic energy is calculated as $W_{\text {kin,e }}=21.18 \mathrm{keV}$. Thus, the accelerated electrons have an energy density of $w_{\mathrm{e}, \mathrm{acc}}=N_{\mathrm{acc}} W_{\text {kin,e }}=4.55 \times$ $10^{-7} \mathrm{erg} \mathrm{cm}^{-3}$. The energy density of the protons inflowing towards the shock can be calculated as $w_{\mathrm{i}, \text { in }}=N_{\mathrm{e}} m_{\mathrm{i}} V_{\mathrm{sh}}^{2} / 2=$ $0.31 \mathrm{erg} \mathrm{\textrm {cm } ^ { - 3 }}$ for $V_{\mathrm{sh}}=1000 \mathrm{~km} \mathrm{~s}^{-1}$. According to the observations at Earth's bow shock (Paschmann et al. 1981), 2\% of this incoming energy is typically transferred into the accelerated protons; i.e., the energy density $w_{\text {i,acc }}$ of the accelerated protons is about $w_{\mathrm{i} \text {,acc }}=6.2 \times 10^{-3} \mathrm{erg} \mathrm{cm}^{-3}$. Thus, $w_{\mathrm{e}, \text { acc }}=0.734 \times 10^{-4} w_{\mathrm{i} \text {,acc }}$; i.e., only a fraction of $\approx 10^{-4}$ of the energy of the accelerated protons is really needed for the electron acceleration. If one assumes that $10 \%$ of the whistler energy is going into the accelerated electrons, the whistler energy den-

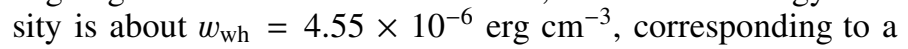
whistler amplitude of $B_{\mathrm{wh}}=0.011 \mathrm{G}$, i.e., $0.011 B_{0}$ (because of $\left.B_{0}=1 \mathrm{G}\right)$. In summary, only a very small fraction $\left(\approx 10^{-6}\right)$ of the shock energy is transferred into the accelerated electrons. 
In order to give some estimation with respect to the acceleration time, Eq. (36) is employed. Adopting $\Delta \bar{W}_{\mathrm{kin}}^{\prime}=0.0414$ (corresponding to an energy of $21.18 \mathrm{keV}), \beta_{\perp}^{\mathrm{e}}=0.2685, V_{\mathrm{ph}}^{\prime}=0.39$ (corresponding to $\omega^{\prime}=0.188$ and $\left.k^{\prime}=0.4815\right), B_{\mathrm{wh}}^{\prime}=0.011$, and $S=20$ as typical values for the accelerated electrons (see above), one gets $t^{\prime}=1130$ and $t_{\text {acc }}=64 \times 10^{-6} \mathrm{~s}$ (because of $\omega_{\text {ce }}=1.758 \times 10^{7} \mathrm{~s}^{-1}$ for $B=1 \mathrm{G}$ ). In summary, the electrons are accelerated within a few tens of microseconds for this special choice of the parameters and the electron velocity components in the resonance band.

The proposed mechanism is only able to explain the generation of energetic electrons in the upstream region. Usually, positively and negatively drifting herringbone structures are observed, as presented in Fig. 1. They are commonly interpreted as electron beams accelerated at the shock and traveling into the up(negative drift) and downstream (positive drift) region (Nelson $\&$ Melrose 1985). But that may not necessarily be true. As seen in Fig. 1, the backbone is located within the range $50-55 \mathrm{MHz}$, corresponding to a height $478-441 \mathrm{Mm}$ above the photosphere by using a twofold Newkirk (1961) model, see Eq. (2). In contrast to that, the herringbones appear in a wider frequency range 40-80 MHz corresponding to a height range of 574-316 Mm above the photosphere; i.e., the herringbone sources cover a wide range in the corona. Spatial radio-heliographic observations have revealed that solar type II radio burst sources mainly propagate nonradially away from active regions (Gopalswamy et al. 1997, 2000; Aurass et al. 1998; Klassen et al. 1999; Klein et al. 1999). Since the shock wave is a spatially extended feature in the corona, the electrons can be accelerated at different sites of the shock. If the shock shows a large-scale curved shape, the upstream region can be directed towards both lower and higher densities, so that the shock accelerated electron beam leads to a negatively and positively drifting signature (i.e., herringbone) in the dynamic radio spectrum. This scenario explains the appearance of negatively and positively drifting herringbones, although the associated beams are generated in the upstream region and traveling away from the shock; see also Fig. 2 in Holman \& Pesses (1983).

The proposed mechanism of electron acceleration is applicable to quasi-perpendicular shocks; i.e., the angle $\theta_{\mathrm{B}, \mathrm{n}}$ is in the range of $50^{\circ}-75^{\circ}$. It explains the production of energetic electrons at shocks, as really observed by spacecraft missions (see Fig. 2). A continuous regime for electron acceleration is achieved, when the electron moves in the resonance band. The proposed mechanism can also act at other shocks in space plasmas, e.g., at supernova remnants.

Acknowledgements. R.M. was supported by Deutscher Akademischer Austauschdienst (DAAD) grant Ref. 324 A/03/10336.

\section{Appendix A: Whistler wave field}

In order to describe the electric and magnetic fields accompanied by the whistler wave, the ambient magnetic field is assumed to be along the $\hat{x}$-axis, i.e., $B_{0}(1,0,0)$. Since whistler waves propagating along the magnetic field are considered, they are not accompanied by an electric field along the magnetic field, i.e., $\boldsymbol{E}_{\mathrm{wh}}=\left(0, E_{y}, E_{z}\right)$. Likewise, all varying quantities are assumed to depend only on the spatial and temporal coordinates $x$ and $t$, respectively. Then, the Ansatz

$B_{y}^{\prime}=B_{\mathrm{wh}}^{\prime} \sin \left[k^{\prime} x^{\prime}-\omega^{\prime} t^{\prime}\right]$

$B_{z}^{\prime}=B_{\mathrm{wh}}^{\prime} \cos \left[k^{\prime} x^{\prime}-\omega^{\prime} t^{\prime}\right]$ describes a righthand, circularly polarized whistler wave in normalized quantities as introduced in Sect. 2. The corresponding electric fields are deduced from the induction equation

$$
\begin{aligned}
& \frac{1}{c} \frac{\partial B_{x}}{\partial t}=0 \\
& \frac{1}{c} \frac{\partial B_{y}}{\partial t}=\frac{\partial E_{z}}{\partial x} \\
& \frac{1}{c} \frac{\partial B_{z}}{\partial t}=-\frac{\partial E_{y}}{\partial x} .
\end{aligned}
$$

It can be written in normalized quantities by

$$
\begin{aligned}
& \frac{1}{S} \frac{\partial B_{y}^{\prime}}{\partial t^{\prime}}=\frac{\partial E_{z}^{\prime}}{\partial x^{\prime}} \\
& \frac{1}{S} \frac{\partial B_{z}^{\prime}}{\partial t^{\prime}}=-\frac{\partial E_{y}^{\prime}}{\partial x^{\prime}}
\end{aligned}
$$

with $S=\omega_{\text {pe }} / \omega_{\text {ce }}$. Substituting the Ansatz (A.1) and (A.2) into Eqs. (A.6) and (A.7) and, subsequently, performing the integration, one can obtain an expression for the electric field of the whistler in terms of its magnetic field,

$$
\begin{aligned}
& E_{y}^{\prime}=\frac{V_{\mathrm{ph}}^{\prime}}{S} B_{\mathrm{wh}}^{\prime} \cos \left[k^{\prime} x^{\prime}-\omega^{\prime} t^{\prime}\right] \\
& E_{z}^{\prime}=-\frac{V_{\mathrm{ph}}^{\prime}}{S} B_{\mathrm{wh}}^{\prime} \sin \left[k^{\prime} x^{\prime}-\omega^{\prime} t^{\prime}\right]
\end{aligned}
$$

with $V_{\mathrm{ph}}^{\prime}=\omega^{\prime} / k^{\prime}$ as the normalized whistler phase velocity. These expressions of the electric and magnetic fields of the whistler waves have been substituted into Eq. (29).

\section{Appendix B: Electron motion in a homogeneous magnetic field}

The motion of an electron in a homogeneous magnetic field along the $\hat{x}$-axis can be expressed in the normalized way (see Sect. 2) by:

$$
\begin{aligned}
\frac{\mathrm{d} p_{x}^{\prime}}{\mathrm{d} t^{\prime}} & =0 \\
\frac{\mathrm{d} p_{y}^{\prime}}{\mathrm{d} t^{\prime}} & =-\frac{p_{z}^{\prime}}{\left(1+p^{\prime 2}\right)^{1 / 2}} \\
\frac{\mathrm{d} p_{z}^{\prime}}{\mathrm{d} t^{\prime}} & =\frac{p_{y}^{\prime}}{\left(1+p^{\prime 2}\right)^{1 / 2}}
\end{aligned}
$$

and

$$
\begin{aligned}
\frac{\mathrm{d} x^{\prime}}{\mathrm{d} t^{\prime}} & =\frac{S}{\left(1+p^{\prime 2}\right)^{1 / 2}} p_{\|} \\
\frac{\mathrm{d} y^{\prime}}{\mathrm{d} t^{\prime}} & =-S p_{\perp} \sin \left(\omega_{\mathrm{ce}}^{\prime} t^{\prime}+\phi_{0}\right) \\
\frac{\mathrm{d} z^{\prime}}{\mathrm{d} t^{\prime}} & =S p_{\perp} \cos \left(\omega_{\mathrm{ce}}^{\prime} t^{\prime}+\phi_{0}\right)
\end{aligned}
$$

according to Eqs. (26) and (27). Here, $\omega_{\mathrm{ce}}^{\prime}$ denotes the relativistically shifted electron gyrofrequency normalized to $\omega_{\text {ce }}$, i.e., $\omega_{\text {ce }}^{\prime}=\gamma^{-1}$ with $\gamma=\left(1-\beta^{2}\right)^{-1 / 2}=\left(1+p^{\prime 2}\right)^{1 / 2}$. The solutions of the above equations of motion are:

$$
\begin{aligned}
& p_{x}^{\prime}=p_{\|}=\text {const. } \\
& p_{y}^{\prime}=-p_{\perp} \sin \left(\omega_{\mathrm{ce}}^{\prime} t^{\prime}+\phi_{0}\right) \\
& p_{z}^{\prime}=p_{\perp} \cos \left(\omega_{\mathrm{ce}}^{\prime} t^{\prime}+\phi_{0}\right)
\end{aligned}
$$


and

$$
\begin{aligned}
x^{\prime}\left(t^{\prime}\right) & =\frac{S}{\left(1+p^{\prime 2}\right)^{1 / 2}} p_{\|} t^{\prime}+x_{0} \\
y^{\prime}\left(t^{\prime}\right) & =S p_{\perp} \cos \left(\omega_{\mathrm{ce}}^{\prime} t^{\prime}+\phi_{0}\right)+y_{0} \\
z^{\prime}\left(t^{\prime}\right) & =S p_{\perp} \sin \left(\omega_{\mathrm{ce}}^{\prime} t^{\prime}+\phi_{0}\right)+z_{0} .
\end{aligned}
$$

These solutions have been used to enter into Eq. (29).

\section{References}

Aurass, H. 1997, in Coronal Physics from Radio and Space Observations, ed. G. Trottet, LNP, 483, 135

Aurass, H., Hofmann, A., \& Urbarz, H.-W. 1998, A\&A, 334, 289

Axford, W. I., Leer, E., \& Skadron, G. 1978, in International Cosmic Ray Conference, 132

Baumjohann, W., \& Treumann, R. A. 1997, Basic space plasma physics (London: Imperial College Press)

Cairns, I. H., \& Robinson, R. D. 1987, Sol. Phys., 111, 365

Classen, H. T., \& Aurass, H. 2002, A\&A, 384, 1098

Classen, H.-T., Mann, G., \& Keppler, E. 1998, A\&A, 335, 1101

Classen, H.-T., Mann, G., Forsyth, R. J., \& Keppler, E. 1999, A\&A, 347, 313

Dulk, G. A., \& McLean, D. J. 1978, Sol. Phys., 57, 279

Fermi, E. 1949, Phys. Rev., 75, 1169

Gopalswamy, N. 2006, Coronal mass ejections and type II radio bursts, Solar Eruptions and Energetic Particles, 207

Gopalswamy, N., \& Kundu, M. R. 1992, ApJ, 390, L37

Gopalswamy, N., Kundu, M. R., Manoharan, P. K., et al. 1997, ApJ, 486, 1036

Gopalswamy, N., Kaiser, M. L., Sato, J., \& Pick, M. 2000, NASA STI/Recon Technical Report N, 32759

Gurnett, D. A., Anderson, R. R., Haeusler, B., Haerendel, G., \& Bauer, O. H. 1985, Geophys. Res. Lett., 12, 851

Holman, G. D., \& Pesses, M. E. 1983, ApJ, 267, 837

Kennel, C. F., Edmiston, J. P., \& Hada, T. 1985, Washington DC American Geophysical Union Geophysical Monograph Series, 34, 1

Kirk, J. G. 1994, in Saas-Fee Advanced Course 24: Plasma Astrophysics, ed. A. O. Benz, \& T. J.-L. Courvoisier, 225

Klassen, A., Aurass, H., Klein, K.-L., Hofmann, A., \& Mann, G. 1999, A\&A, 343,287
Klein, K.-L., Khan, J. I., Vilmer, N., Delouis, J.-M., \& Aurass, H. 1999, A\&A, 346, L53

Koutchmy, S. 1994, Advances in Space Research, 14, 29

Lara, A., Gopalswamy, N., Nunes, S., Muñoz, G., \& Yashiro, S. 2003, Geophys. Res. Lett., 30, 4

Mann, G. 1995, in Coronal Magnetic Energy Releases, ed. A. O. Benz, \& A. Krüger, LNP, 444, 183

Mann, G. 2006, EIT waves and coronal shock waves, Solar Eruptions and Energetic Particles, 221

Mann, G., \& Klassen, A. 2005, A\&A, 441, 319

Mann, G., Aurass, H., Voigt, W., \& Paschke, J. 1992, in Coronal Streamers, Coronal Loops, and Coronal and Solar Wind Composition, ed. C. Mattok, ESA SP-348, 129

Mann, G., Classen, T., \& Aurass, H. 1995, A\&A, 295, 775

Mann, G., Jansen, F., MacDowall, R. J., Kaiser, M. L., \& Stone, R. G. 1999, A\&A, 391, 749

Mann, G., Classen, H. T., Keppler, E., \& Roelof, E. C. 2002, A\&A, 348, 614

Melrose, D. B. 1985, Plasma emission mechanisms, Solar Radiophysics: Studies of Emission from the Sun at Metre Wavelengths, 177

Nelson, G. J., \& Melrose, D. B. 1985, type II bursts, Solar Radiophysics: Studies of Emission from the Sun at Metre Wavelengths, 333

Newkirk, G. J. 1961, ApJ, 133, 983

Paschmann, G., Sckopke, N., Asbridge, J. R., Bame, S. J., \& Gosling, J. T. 1980, J. Geophys. Res., 85, 4689

Paschmann, G., Sckopke, N., Papamastorakis, I., et al. 1981, J. Geophys. Res., 86,4355

Priest, E. R. 1982, Solar magneto-hydrodynamics (Dordrecht, Holland; Boston: D. Reidel Pub. Co.; Hingham)

Schlickeiser, R. 1984, A\&A, 136, 227

Scholer, M., Mann, G., Chalov, S., et al. 1999, Space Sci. Rev., 89, 369

Stewart, R. T., McCabe, M. K., Koomen, M. J., Hansen, R. T., \& Dulk, G. A. 1974a, Sol. Phys., 36, 203

Stewart, R. T., Howard, R. A., Hansen, F., Gergely, T., \& Kundu, M. 1974b, Sol. Phys., 36, 219

Uchida, Y., Altschuler, M. D., \& Newkirk, G. J. 1973, Sol. Phys., 28, 495

Vocks, C., \& Mann, G. 2006, A\&A, 452, 331

Vrsnak, B., Ruzdjak, V., Zlobec, P., \& Aurass, H. 1995, Sol. Phys., 158, 331

Wild, J. P., \& McCready, L. L. 1950, Australian J. Sci. Res. A Phys. Sci., 3, 387

Winske, D., \& Leroy, M. M. 1984, J. Geophys. Res., 89, 2673

Zlobec, P., Messerotti, M., Karlicky, M., \& Urbarz, H. 1993, Sol. Phys., 144, 373 